\title{
A POINTWISE REPRESENTATION OF THE S-MATRIX KOHN VARIATIONAL PRINCIPLE FOR QUANTUM SCATTERING
}

\author{
Andrew C. PEET and William H. MILLER \\ Department of Chemistry, University of California, \\ and Materials and Chemical Sciences Division, Lawrence Berkeley Laboratory, Berkeley, C.A 94720, US.4
}

Received 2 June 1988

\begin{abstract}
A method is proposed for reducing the complexity of scattering calculations carried out using the Kohn variational principle. The technique is based upon the use of a pointwise representation for the $L^{2}$ part of the basis set and eliminates the need to explicitly evaluate any integrals involving such functions. Application to potential and inelastic scattering test cases show the method to be of good accuracy.
\end{abstract}

The $S$-matrix version of the Kohn variational principle [1,2] has recently enjoyed much success in describing scattering processes of both electrons [3] and heavy particles [2,4-6]. In particular, the method has been shown to provide a powerful description of the full 3D reactive scattering event, with studies of $\mathrm{H}+\mathrm{H}_{2}[5]$ and $\mathrm{F}+\mathrm{H}_{2}$ [6] having been reported. These calculations and other related works [7] represent a significant advance in our understanding of elementary chemical reactions.

Calculation of the $S$-matrix in the Kohn formalism may be considered in two parts. First, the evaluation of matrix elements of the Hamiltonian over a basis set of scattering and $L^{2}$ functions and, second, the algebraic manipulation of these matrices to give the $S$-matrix. Formally, the setting up of the matrices is a lower-order computational process than the matrix manipulations and so the latter will dominate the effort required for a sufficiently large basis. However, for the problems presently being studied, the size of basis set and the nature of the integrals dictate that the calculation of the matrices constitutes a considerable fraction of the effort involved.

Here we present a method which greatly simplifies the application of the Kohn principle by avoiding the need to explicitly evaluate any integrals over the $L^{2}$ basis set. The technique is based, initially, upon an $N$-point quadrature approximation to the integrals, where $N$ is the number of $L^{2}$ functions. After expressing the integrals as products of square matrices, simple matrix manipulations then allow the Kohn expression to be reduced to a form in which no quadratures over the $L^{2}$ basis functions are present. The final expression is found to have a similar structure to the original one except that the matrices involving the $L^{2}$ basis now occur in point space.

This study constitutes a continuation of the recent interest in the use of pointwise methods to reduce the computational complexity of large quantum mechanical calculations. These techniques are broadly based upon the premise that a minimum of $N$ potential evaluations is required to uniquely determine a wavefunction expanded in terms of $N$ basis functions. Although such methods have existed for many years [8,9], it is only recently that they have met with much popularity and been shown to provide an accurate description of real quantum mechanical systems. Most relevantly, Light and co-workers have developed a version of the technique which they call the discrete variable representation and successfully applied it to both scattering $[10,11]$ and bound state regions [12-14]. Furthermore, Friesner [15-17] has managed to make great savings in the computation of electronic energies by a scheme which he terms the pseudospectral representation.

We begin the presentation of the technique as applied to the Kohn principle by introducing the formalism to be used and obtaining an expression for the case of s-wave potential scattering. The method is illustrated 
by applying it to a model electron scattering problem which is well described by functions for which a Gaussian quadrature scheme exists. In section 3 more general basis sets and quadrature schemes are considered, and we also address the problem of hermiticity of the Hamiltonian matrix when calculated by an approximate quadrature. Application to a heavy particle potential scattering problem shows that the technique provides an accurate description when compared with the method in which all integrals are evaluated exactly. The extension to multidimensional systems is discussed in section 4 and the model $\mathrm{He}-\mathrm{H}_{2}$ vibrational energy transfer problem of Secrest and Johnson [18] is investigated, with correspondingly successful results.

\section{Basic formalism}

We first consider s-wave potential scattering for which all the essential features of the pointwise method may be illustrated. The Hamiltonian has the standard form

$H=-\frac{\hbar^{2}}{2 \mu} \frac{\mathrm{d}^{2}}{\mathrm{~d} r^{2}}+V(r)$,

with $V(r) \rightarrow 0$ as $r \rightarrow \infty$. The Kohn variational expression for the $S$-matrix is then [2]

$$
S=\frac{\mathrm{i}}{\hbar}\left(B-\frac{C^{2}}{B^{*}}\right) \text {, }
$$

where the complex quantities $B$ and $C$ are given by

$$
\begin{aligned}
& B=\left\langle u_{0}|H-E| u_{0}\right\rangle-\sum_{l, l^{\prime}=1}^{N}\left\langle u_{l}|H-E| u_{0}\right\rangle\left(\left\langle u_{l}|H-E| u_{l^{\prime}}\right\rangle\right)^{-1}\left\langle u_{l^{\prime}}|H-E| u_{0}\right\rangle, \\
& C=\left\langle u_{0}^{*}|H-E| u_{0}\right\rangle-\sum_{l, l^{\prime}=1}^{N}\left\langle u_{l}|H-E| u_{0}\right\rangle^{*}\left(\left\langle u_{l}|H-E| u_{l^{\prime}}\right\rangle\right)^{-1}\left\langle u_{l^{\prime}}|H-E| u_{0}\right\rangle .
\end{aligned}
$$

It should be noted that throughout this paper functions inside a bra symbol are not complex conjugated as is usually the case; any complex conjugation (as, e.g., in eq. (3b)) is indicated explicitly. The function $u_{0}(r)$ is an incoming radial wave multiplied by a cut-off function $f(r)$ to regularise it at small $r$. The real basis functions $u_{l}(r), l=1, \ldots, N$ are square integrable and form the major part of the basis set.

From eq. (3) we see that there are two main demands on the computational effort. First, the setting up of the matrices which involve the real basis functions and second, the inversion of the large square matrix constructed from these functions. In this paper we address the former of these two problems. The strategy is to reduce the integrals over the real basis functions to minimal $N$-point quadratures. Choosing the points $\left\{\boldsymbol{r}_{i}\right\}$ and corresponding weights $\left\{w_{i}\right\}$ we define the matrices

$$
\begin{aligned}
& R_{i l}=\sqrt{w_{i}} u_{l}\left(r_{i}\right), \quad V_{i i^{\prime}}=\delta_{i i^{\prime}} V\left(r_{i}\right), \quad D_{i l}=-\frac{\hbar^{2}}{2 \mu} \sqrt{w_{i}} u_{l}^{\prime \prime}\left(r_{i}\right), \\
& \left(U_{0}\right)_{i}=\sqrt{w_{i}} u_{0}\left(r_{i}\right), \quad\left(D_{0}\right)_{i}=-\frac{\hbar^{2}}{2 \mu} \sqrt{w_{i}} u_{0}^{\prime \prime}\left(r_{i}\right),
\end{aligned}
$$

where the prime denotes differentiation with respect to $r$. These quantities may then be used to write $B$ and $C$ in the (approximate) forms

$$
\begin{aligned}
& B=\left\langle u_{0}|H-E| u_{0}\right\rangle-\left(\mathbf{R}^{\mathrm{T}} \mathbf{M}_{0}\right)^{\mathrm{T}}\left(\mathbf{R}^{\mathrm{T}} \mathbf{M R}\right)^{-1}\left(\mathbf{R}^{\mathrm{T}} \mathbf{M}_{0}\right), \\
& C=\left\langle u_{0}^{*}|H-E| u_{0}\right\rangle-\left(\mathbf{R}^{\mathrm{T}} \mathbf{M}_{0}^{*}\right)^{\mathrm{T}}\left(\mathbf{R}^{\mathrm{T}} \mathbf{M R}\right)^{-1}\left(\mathbf{R}^{\mathrm{T}} \mathbf{M}_{0}\right),
\end{aligned}
$$

where the superscript $T$ denotes the matrix transpose and 


$$
\begin{aligned}
& \mathbf{M}_{0}=\mathbf{D}_{0}+(\mathbf{V}-E \mathbf{I}) \mathbf{U}_{0}, \\
& \mathbf{M}=\mathbf{T}+\mathbf{V}-\mathbf{E} \mathbf{l} .
\end{aligned}
$$

The kinetic energy matrix $T$ is given by

$\mathbf{T}=\mathbf{D} \mathbf{R}^{-1}$.

The two free-free matrix elements in eq. (5), $\left\langle u_{0}|H-E| u_{0}\right\rangle$ and $\left\langle u_{0}^{*}|H-E| u_{0}\right\rangle$, are assumed to be evaluated exactly.

Since all the matrices involved are square, it is not hard to show that the matrix $\mathbf{R}$ completely cancels out of eq. (5) leaving the simple expressions

$$
\begin{aligned}
& B=\left\langle u_{0}|H-E| u_{0}\right\rangle-\mathbf{M}_{0}^{\mathrm{T}} \mathbf{M}^{-1} \mathbf{M}_{0}, \\
& C=\left\langle u_{0}^{*}|H-E| u_{0}\right\rangle-\mathbf{M}_{0}^{* \mathrm{~T}} \mathbf{M}^{-1} \mathbf{M}_{0} .
\end{aligned}
$$

Due to the absence of $\mathbf{R}$, the final expressions for $B$ and $C$ no longer contain any quadratures over the real basis functions. Furthermore, since $\mathbf{R}$ is a transformation matrix from the basis set to the pointwise representation, the $L^{2}$ space now appears only in a pointwise fashion. However, it should be noted that since eqs. (5) and (8) are equivalent, the pointwise method will give identical results to the basis set approach with the integrals evaluated by the $N$-point quadrature. The accuracy of the pointwise representation compared with the full variational method is thus determined by the errors introduced from the non-exact quadrature. If these errors are not significantly greater than those due to the truncation of the basis set, then the pointwise and variational approaches will be of comparable accuracy.

From eqs. (6b) and (7) we see that the $L^{2}$ basis functions now appear only in the expression for the pointwise kinetic energy matrix $T$. The potential matrix is diagonal in point space with its elements being merely the potential evaluated at the quadrature points. The problem of constructing the potential matrix in the basis set representation has thus largely been replaced by one of evaluating the kinetic energy matrix in the pointwise representation. However, as we shall see in section 5, this is often a much simpler task.

\section{Application to $1 \mathrm{D}$ electron scattering}

In order to test the accuracy of this pointwise representation of the Kohn expression we first consider the electron scattering problem used by Staszewska and Truhlar [19]. The potential is attractive and has the form $V(r)=-\exp (-r)$.

The scattering function $u_{0}(r)$ is taken to have the form of section 2 with the cut-off function $f(r)=1-\exp (-\alpha r)$. The $L^{2}$ basis set is the same as used previously [2],

$u_{l}(r)=N_{l} r^{l-1} \exp (-\alpha r), \quad l=1, \ldots, N$.

These real functions are linear combinations of Gauss-Laguerre polynomials multiplied by the conventional weighting functions and so a Gaussian quadrature scheme exists for choosing the weights and points. This scheme evaluates the kinetic energy matrix exactly since the second derivative of eq. (10) is a linear combination of the basis functions and the Gaussian quadrature evaluates all overlap integrals exactly.

Table 1 shows how the error in the tangent of the phase shift varies with size of the real basis for the pointwise method and compares the results with those obtained by evaluating all the integrals exactly, the variational method. It is seen that the pointwise results converge with an accuracy comparable to those of the variational calculations. This clearly demonstrates the potential of the method to reduce the complexity of the calculation without leading to a significant increase in the basis set size. Actually, the basis set required to obtain good 
Table 1

Fractional errors [4] in $\tan \delta$ for $1 D$ electron scattering test case. The scattering energy is given by $k=0.55$ au [2] and $\alpha=2.5$. The exact result is $\tan \delta=2.2003827$

\begin{tabular}{cccccc}
\hline$N$ & Variational & Pointwise & $N$ & Variational & Pointwise \\
\hline 2 & -0.0001 & +0.1111 & 5 & -0.0003 & -0.0010 \\
3 & -0.0019 & -0.0013 & 6 & -0.0001 & -0.0005 \\
4 & -0.0011 & -0.0013 & & & \\
\hline
\end{tabular}

results in this test problem is so small that it is unwise to extrapolate such conclusions to larger systems. Also, many problems are not conveniently described by a set of functions for which a Gaussian quadrature is available. It is, therefore, important to consider the extension to a more adaptable basis and non-Gaussian quadratures.

\section{Combining the pointwise description with local basis functions}

Perhaps the most adaptable of the basis sets presently available for describing one-dimensional motion is the distributed Gaussian basis proposed by Hamiltonian and Light [20],

$u_{l}(r)=\left(\frac{2 A_{l}}{\pi}\right)^{1 / 4} \exp \left[-A_{l}\left(r-r_{l}\right)^{2}\right], \quad l=1, \ldots, N$.

Since a single Gaussian quadrature scheme does not exist for this basis set, other prescriptions for choosing the points and weights must be sought. Perhaps the most obvious place to put the points is at the centres of the Gaussians. The weights are then most conveniently taken to be those of a trapezoidal rule quadrature, where it is undoubtedly best to assume that the end points of the quadrature scheme lie outside the $N$-point range.

The use of such a simple quadrature scheme dictates that the kinetic energy matrix will no longer be evaluated exactly over the basis set. More importantly, the matrix will in general not be symmetric, leading to an unsymmetric form of the pointwise matrix $\mathrm{T}$. Here we avoid this problem by rewriting the kinetic energy matrix in an inherently symmetric form. Integrating once by parts we obtain the identity

$-\frac{h^{2}}{2 \mu} \int u_{l}(r) u_{l^{\prime}}^{\prime \prime}(r) \mathrm{d} r=\frac{h^{2}}{2 \mu} \int u_{l}^{\prime}\left(r_{l}\right) u_{l^{\prime}}^{\prime}(r) \mathrm{d} r$,

where the prime denotes differentiation with respect to $r$. The integral on the right-hand side of this expression may be integrated by any conventional quadrature scheme to yield a symmetric matrix. The expression for the matrix $\mathbf{T}$ in the pointwise space then becomes

$\mathbf{T}=\frac{\hbar^{2}}{2 \mu}\left(\mathbf{d} \mathbf{R}^{-1}\right)^{\mathrm{T}} \mathbf{d} \mathbf{R}^{-1}$,

where the matrix $\mathbf{d}$ is defined by

$d_{i l}=\sqrt{w_{i}} u_{i}^{\prime}\left(r_{i}\right)$.

In practice, a reasonable quadrature scheme will evaluate the kinetic energy matrix quite accurately and so it could be used without further adaptation. However, writing $T$ in a truly symmetric form has the computational advantage of reduced storage and matrix inversion time as well as the conceptual nicety of preserving the numerical hermiticity of the Hamiltonian over the $L^{2}$ basis. This technique for ensuring hermiticity is not, of course, unique. An alternative method, favoured by Light [12], is to calculate the kinetic energy matrix exactly in the basis set representation and then transform to the pointwise scheme using the matrix $\mathbf{R}$. This 
Table 2

Fractional errors in $\tan \delta$ for $\mathrm{He}-\mathrm{H}_{2}$ elastic scattering. The parameters used were $E=6.0, \alpha_{1}=0.5, R_{1}=10.0, r_{\min }=1.0, r_{\max }=20.0$ and $\mu=0.667$, all in the reduced units of Secrest and Johnson [18]. The exact value for $\tan \delta$ is 0.750188

\begin{tabular}{cccccc}
\hline$N$ & Variational & Pointwise & $N$ & Variational & Pointwise \\
\hline 16 & -0.0717 & -0.5581 & 22 & -0.0015 & -0.0100 \\
18 & -0.0071 & -0.2758 & 24 & -0.0004 & -0.0005 \\
20 & -0.0037 & -0.0647 & 26 & -0.0001 & +0.0006 \\
\hline
\end{tabular}

technique has also been implemented and was found to yicld comparable accuracy for the examples presented below.

The procedure outlined above was tested out on the elastic part of the $\mathrm{He}-\mathrm{H}_{2}$ scattering problem of Secrest and Johnson [18], The intermolecular potential for this system has the form

$V(r)=V_{0} \exp (-\gamma r)$,

with the parameters $V_{0}=12.0$ au and $\gamma=0.315$ au. The cut-off function $f(r)$ used to regularise the scattering basis function $u_{0}$ was

$f(r)=\frac{1}{2}\left\{1+\tanh \left[\alpha_{1}\left(r-r_{1}\right)\right]\right\}$,

which has been used in previous calculations [4]. The Gaussians were distributed evenly between the values 1.0 and $20.0 a_{0}$ in $r$ while the exponents of the Gaussians were chosen by the prescription

$A_{i}=\frac{c^{2}}{\Delta r^{2}}$

where $\Delta r$ is the distance between the centres of the Gaussians and $c$ is a parameter which may be varied.

The results are given in table 2 , where they are also compared with the full variational calculations. The parameter $c$ was varied and found to be approximately optimum at 0.75 and 0.5 for the variational and pointwise schemes, respectively. It should be noted that broader Gaussians are used in the pointwise method. This is as expected on the grounds of increasing the accuracy of the trapezoidal rule quadrature.

From table 2 it is seen that the pointwise method requires a basis set about $20 \%$ greater than the variational method to attain results with a $1 \%$ error. This result is very encouraging especially when we consider the simple minded nature in which the points and weights were chosen. Furthermore, if greater accuracy is desired then fewer extra functions are required by the pointwise technique. Indeed, the size of basis which gives results correct to $0.1 \%$ is the same for both methods.

\section{Multidimensional problems}

The extension of the pointwise representation to systems with more than one degree of freedom is straightforward and so we simply summarise the basic formulae. The Kohn expression now takes the form [2]

$\mathbf{S}=\frac{\mathrm{i}}{h}\left(\mathbf{B}-\mathbf{C}^{\mathrm{T}} \mathbf{B}^{*-1} \mathbf{C}\right)$

where $\mathbf{B}$ is a complex matrix which is the generalisation of eq. (3a),

$$
\begin{aligned}
B_{n n^{\prime}} & =\left\langle u_{0} \phi_{n}|H-E| u_{0} \phi_{n^{\prime}}\right\rangle \\
- & \sum_{l, l^{\prime}=1}^{N} \sum_{m . m^{\prime}=1}^{M}\left\langle u_{l} \phi_{m}|H-E| u_{0} \phi_{n}\right\rangle\left(\left\langle u_{l} \phi_{m}|H-E| u_{l} \phi_{m^{\prime}}\right\rangle\right)^{-1}\left\langle u_{l^{\prime}} \phi_{m^{\prime}}|H-E| u_{0} \phi_{n^{\prime}}\right\rangle
\end{aligned}
$$


and $\mathbf{C}$ is the corresponding generalisation of eq. (3b). The quantities $\phi_{n}(q), n=1, \ldots, M$ are basis functions associated with the internal coordinates q. For the open channels, which the indices $n$ and $n^{\prime}$ are restricted to, these are asymptotic eigenfunctions.

A transformation matrix $\mathbf{R}$ may be defined analogous to the one used in the potential scattering case. Choosing the weights $\left\{\boldsymbol{w}_{i}\right\}$ and $\left\{\boldsymbol{W}_{j}\right\}$ associated with the points $\left\{\boldsymbol{r}_{i}\right\}$ and $\left\{\boldsymbol{q}_{j}\right\}$, respectively, we obtain

$R_{i j, l n}=\sqrt{w_{i}} \sqrt{W_{j}} u_{i}\left(r_{i}\right) \phi_{n}\left(\boldsymbol{q}_{j}\right)$.

The most direct extension of the one-dimensional case is obtained if the kinetic energy operator is taken to be the complete Hamiltonian $H$ with the intermolecular potential removed. $\mathrm{D}$ and $\mathrm{D}_{n}$ are then simply defined in terms of the basis functions under the action of the kinetic energy operator, evaluated at the quadrature points. With these quantities, the derivation of the pointwise expression for the multidimensional case follows closely the form presented in section 2.

An important feature of multidimensional systems is that several simplifications may now be implemented. Firstly, we may construct the inverse of the transformation matrix from the inverses of the matrices in each dimension. This is a crucial result since it avoids the need to explicitly invert a matrix of dimension equal to the size of the $L^{2}$ basis. Furthermore, if the kinetic energy operator can be expressed as a sum of several terms, then the kinetic energy matrix may be evaluated as a sum of corresponding matrices. This can provide a great simplification since it is common for many of the terms in the kinetic energy operator of a real system to contain only a subset of the variables. Such structure allows the kinetic energy matrix to be constructed from smaller matrices which have been evaluated in a space of lower dimension.

These properties are best illustrated by a simple example. We consider the collinear vibrational energy transfer problem of Secrest and Johnson [18]. The Hamiltonian for this system may be written as

$H=t_{r}(r)+h_{q}(q)+V(r, q)$,

where $t_{r}(r)$ is the kinetic energy operator for the collision, $h_{q}(q)$ is the vibrational Hamiltonian of the diatom and $V(r, q)$ is the interaction potential. The kinctic encrgy matrix in the basis set representation is the sum of matrices arising from $t_{r}$ and $h_{q}$. Since the two operators each depend upon only one of the variables, these matrices are direct products of the one-dimensional kinetic energy and overlap matrices. Transforming to the pointwise scheme thus gives

$(T)_{i k, i^{\prime} k^{\prime}}=T_{i i^{\prime}}^{r} \delta_{k k^{\prime}}+\delta_{i i^{\prime}} H_{k k^{\prime}}^{q^{\prime}}$

where $T_{i i^{\prime}}^{r}$ and $H_{k^{\prime}}^{q^{\prime}}$ are the pointwise matrices of the operators $t_{r}$ and $h_{q}$ constructed (as outlined in section 2 ) in the one-dimensional spaces $r$ and $q$, respectively.

In order to test the accuracy of the pointwise method for a multidimensional problem we calculated inelastic transition probabilities for the $\mathrm{He}-\mathrm{H}_{2}$ problem. The interaction potential for this model system has the form

$$
V(r, q)=V_{0} \exp [-\gamma(r-q)] \text {, }
$$

where the parameters $V_{0}$ and $\gamma$ have the values 12.0 and 0.315 atomic units, respectively. The details of the translational basis set are as given in section 4 . The $\mathrm{H}_{2}$ vibrational motion is conveniently described by simple harmonic oscillator functions allowing Gauss-Hermite points and weights to be used. The calculations were carried out at an energy of 6.0 in the reduced units of Secrest and Johnson [18], leading to three open channels.

Results of the calculations are given in table 3. The pointwise scheme requires an increase of about $20 \%$ in the radial basis over the vibrational method to give convergence of the largest inelastic transition probability $\left(P_{01}\right)$ to $1 \%$. The case appears to be more favourable for the smaller probabilities. The convergence with respect to the vibrational basis of the $\mathrm{H}_{2}$ molecule was found to be rapid and similar for both the variational and pointwise methods. This is as expected for a basis which has an associated Gaussian quadrature scheme.

Finally, we note that the only integrals which must be evaluated in the present formulation are the ones be- 
Table 3

Fractional errors in the vibrational inelastic transition probabilities for the model $\mathrm{He}-\mathrm{H}_{2}$ problem. The parameters used are the same as for the $1 \mathrm{D}$ case of table 2 and five $\mathrm{H}_{2}$ vibrational functions are used. The correct transition probabilities are $P_{01}=0.30058(-1)$, $P_{02}=0.11273(-4)$ and $P_{12}=0.14723(-2)$ [4]. Fractional errors with a magnitude greater than 10 are denoted by a dash

\begin{tabular}{|c|c|c|c|c|c|c|}
\hline \multirow[t]{2}{*}{$N$} & \multicolumn{2}{|l|}{$P_{01}$} & \multicolumn{2}{|l|}{$P_{02}$} & \multicolumn{2}{|l|}{$P_{12}$} \\
\hline & variational & pointwise & variational & pointwise & variational & pointwise \\
\hline 12 & -1.664 & - & +1.167 & - & 0.232 & +0.106 \\
\hline 15 & -0.023 & -0.698 & -0.029 & -0.845 & -0.018 & -0.387 \\
\hline 18 & -0.003 & -0.051 & +0.004 & -0.002 & -0.002 & +0.039 \\
\hline 21 & +0.004 & -0.018 & +0.014 & +0.025 & 0.000 & -0.005 \\
\hline 24 & +0.003 & +0.003 & +0.019 & +0.015 & +0.003 & +0.002 \\
\hline
\end{tabular}

tween the scattering basis functions. Due to the oscillatory nature of the integrands, the values of these matrix elements are often very small. This is especially the case when the cut-off function takes effect far out in the region of weak interaction. To test the significance of this we changed the cut-off parameters to $r_{1}=15.0$ and $\alpha_{1}=1 / 3$. With these values, all matrix elements of the type $\left\langle u_{0} \phi_{n}|H-E| u_{0} \phi_{n^{\prime}}\right\rangle$ could be neglected without changing the third figure of the inelastic transition probability.

\section{Conclusions}

A method has been proposed for calculating the $S$-matrix via a pointwise representation of the Kohn variational expression. Use of this scheme eliminates the need to explicitly carry out any integrals which involve $L^{2}$ basis functions. In previous formulations, the calculation of such integrals requires much computational effort since the potential function must usually be evaluated at many points. In contrast, the pointwise method requires the potential to be evaluated only at the same number of points as there are basis functions.

The method was implemented and found to be of comparable accuracy to the full variational form for degrees of freedom which are well described by functions for which a Gaussian quadrature scheme exists. The application to more general situations has also been considered. In this case, a basis set of distributed Gaussians and a simple trapezoidal rule quadrature scheme was found to require no more than a $20 \%$ increase in the size of the basis set to obtain convergence.

Extension of the technique to multidimensional systems was seen to yield some important simplifications in constructing the kinetic energy matrix. Furthermore, the application to a model $\mathrm{He}-\mathrm{H}_{2}$ system showed that the convergence properties were as expected from the one-dimensional calculations. It thus appears that the method should work well for any inelastic scattering process.

In this paper we have applied the pointwise method only to non-reactive processes. However, the real interest in basis set methods for scattering lies in their ability to describe rearrangement collisions, for which exchange interactions [21] are present. The pointwise scheme should be readily applicable to such systems and, if found to be of good accuracy, will be a powerful technique for calculating reaction rates.

\section{Acknowledgement}

This work was supported by the National Science Foundation, grant CHE8416345. ACP gratefully acknowledges the generous support of an SERC/NATO fellowship. 


\section{References}

[1] W. Kohn, Phys. Rev. 74 (1978) 1763.

[2] J.Z.H. Zhang, S.-I. Chu and W.H. Miller, J. Chem. Phys. 88 (1988) 6233.

[3] C.W. McCurdy, T.N. Resigno and B.I. Schneider, Phys. Rev. A 36 (1987) 2061.

[4] W.H. Miller and B.M.D.D. Jansen op der Haar, J. Chem. Phys. 86 (1987) 6213.

[5] J.Z.H. Zhang and W.H. Miller, Chem. Phys. Letters 140 (1987) 329.

[6] J.Z.H. Zhang and W.H. Miller, J. Chem. Phys. 88 (1988) 4549.

[7] K. Haug, D.W. Schwenke, Y. Shima, D.G. Truhlar, J.Z.H. Zhang and D.J. Kouri, J. Phys. Chem. 90 (1986) 6757.

[8] B.A. Finlayson, The method of weighted residuals and variational principles (Academic Press, New York, 1972).

[9] D.O. Harris, G.G. Engerholm and W.D. Gwinn, J. Chem. Phys. 43 (1965) 1515.

[10] J.V. Lill, G.A. Parker and J.C. Light, Chem. Phys. Letters 89 (1982) 483.

[11] R.W. Heather and J.C. Light, J. Chem. Phys. 79 (1983) 147.

[12] J.C. Light, I.P. Hamilton and J.V. Lill, J. Chem. Phys. 82 (1985) 1400.

[13] Z. Bacic and J.C. Light, J. Chem. Phys. 85 (1986) 4594.

[14] Z. Bacic and J.C. Light, J. Chem. Phys. 86 (1987) 3065.

[15] R. Friesner, Chem. Phys. Letters 116 (1985) 39.

[16] R. Friesner, J. Chem. Phys. 85 (1986) 1462.

[17] R. Friesner, J. Chem. Phys. 86 (1987) 3522.

[18] D. Secrest and B.R. Johnson, J. Chem. Phys. 45 (1966) 4556.

[19] G. Staszewska and D.G. Truhlar, Chem. Phys. Letters 130 (1986) 341.

[20] I.P. Hamilton and J.C. Light, J. Chem. Phys. 84 (1986) 306.

[21] W.H. Miller, J. Chem. Phys. 50 (1969) 407. 